\title{
Für einen besseren Workflow im Praxisalltag
}

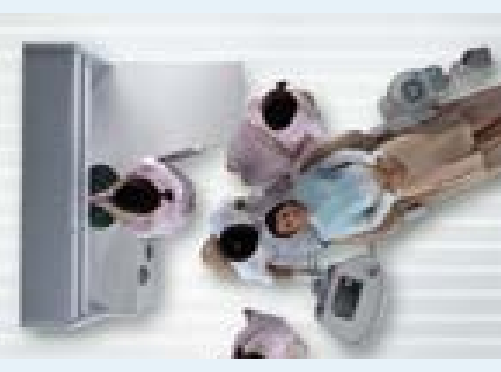

Die aktuelle Spaceline EMCIA des Unternehmens Morita spiegelt den dentalen

Trend zur WorkflowOptimierung. Grundlage der Behandlungseinheit ist das Konzept von Dr. Daryl R. Beach, das seit den 1960er Jahren erfolgreich angewendet wird. Die Einheit bietet maßgeschneiderte Lösungen - orientiert an seinem Konzept. Je nach Bedarf kann der Anwender sein Arbeitsumfeld völlig individuell gestalten und sich zwischen einer Vielzahl an flexiblen Ablagesystemen entscheiden: Zur Auswahl stehen in die Rückenlehne des Patientenstuhls integrierte Instrumente, ein rückenlehnenmontiertes Tray oder ein schwenkbares Arztelement. Weitere Ablagemöglichkeiten bietet ein deckenmontiertes, frei schwenkbares Zusatztray. Alle wichtigen Instrumente und Bedienfunktionen sind dabei so angeordnet, dass sie äußerst komfortabel zu erreichen sind. Die in die Rückenlehne integrierten Instrumente sichern außerdem ein besonders intuitives Handling im Behandlungsalltag. Sie sind sowohl aus der Behandler- als auch aus der Assistenzposition heraus schnell und bequem greifbar und dadurch sofort einsatzbereit. Von den Vorteilen dieses Konzepts können sich Interessierte auch 2016 wieder in den Ergonomie-Fortbildungskursen von Morita überzeugen. Experten vermitteln in kleinen Gruppen die Vorteile einer richtigen Körperhaltung bei der Behandlung. Wer an der Teilnahme interessiert ist, kann sich schon jetzt einen der Termine für 2016 vormerken:

\section{„Präzision ist eine Haltung - Ergonomie und Workflow in der Zahnarztpraxis“}

01.-02. Juli und 14.-15. Oktober 2016 mit Dr. Wolf Neddermeyer (Morita Showroom, Justus-von-Liebig-Str. 26, 63128 Dietzenbach); 28.-29. Oktober 2016 in der Praxis Zahnarzt Sivan Ates (Gotenring 1, 50679 Köln); 25.-26. November 2016 in der Praxis Dr. Tom Schloss \& Dr. Jürgen Wollner (Kornmarkt 8, 90402 Nürnberg).

Nach einer Pressemitteilung der J. Morita Europe GmbH, Dietzenbach www.morita.com/europe. 